\title{
NUMERICAL SIMULATIONS OF BREAKING SOLITARY WAVES
}

\author{
Pierre Lubin $^{1}$ and Stéphane Glockner ${ }^{2}$
}

\begin{abstract}
This paper presents the application of a parallel numerical code to breaking solitary waves impacting a seawall structure. The three-dimensional Navier-Stokes equations are solved in air and water, coupled with a subgrid scale model to take turbulence into account. We compared three numerical methods for the free-surface description, using the classical VOFPLIC and VOF-TVD methods, and an original VOF-SM method recently developed in our numerical tool (Vincent et al. 2010). Some experimental data for solitary waves impinging and overtopping coastal structures are available in literature (Hsiao and Lin, 2010). Solitary waves are often used to model tsunami behaviors because of their hydrodynamic similarities. From a numerical point of view, it allows shorter CPU time simulations, as only one wave breaks. Here we apply the model to simulate three-dimensional solitary waves and compare qualitatively our results with the experimental data. We investigate three configurations of solitary waves impinging and overtopping an impermeable seawall on a 1:20 sloping beach.
\end{abstract}

Keywords: Navier-Stokes; numerical simulation; solitary wave; two-phase flow; breaking waves; air entrainment

\section{INTRODUCTION}

Up to date studies proved the importance of air entrainment for turbulence generation in breaking waves. But, as introduced by Ting (2008) concerning numerical results of three-dimensional flow structures under breaking waves, "the forms and evolution of large eddies obtained in numerical simulations may depend critically on how the free-surface deformation is modeled". Numerical simulation of breaking waves is still a very challenging aim to achieve since small interface deformations, air entrainment and vorticity generation are involved during the overturning and the subsequent impact of the wave.

Existing numerical models still cannot describe wave breaking satisfactorily, considering the air and water mixing areas where a broad range of relevant length and time scales is involved, making it extremely complicated to investigate experimentally and numerically. It is thus very important to use appropriate and accurate numerical schemes for the interface description. Moreover, performing numerical simulations of breaking waves still remains difficult as it requires a large number of mesh grid points, robust and accurate numerical methods, and long CPU time calculations to compute the hydrodynamics from the largest to the smallest length and time scales (Lubin et al. 2011).

The scope of this paper is to show the results obtained for simulating three-dimensional solitary waves shoaling and breaking over a sloping beach with a seawall structures, using a recent original numerical method for simulating free-surface flows (Vincent et al. 2010). The ability of this method to deal with the free-surface deformations will be shown.

\section{MODEL AND NUMERICAL METHODS}

We solve the Navier-Stokes equations in air and water, coupled with a subgrid scale turbulence model. The numerical tool is well suited to deal with strong interface deformations occurring during wave breaking, for example, and with turbulence modeling in the presence of a free-surface in a more general way.

\section{Governing equations}

An incompressible multiphase phase flow between non-miscible fluids can be described by the Navier-Stokes equations in their multiphase form. The governing equations for the Large Eddy Simulation (LES) of an incompressible fluid flow are classically derived by applying a convolution filter to the unsteady Navier-Stokes equations. In the single fluid formulation of the problem, a phase function $C$, or "color" function, is used to locate the different fluids standing $C=O$ in the outer medium, $C=1$ in the considered medium. The interface between two media is repaired by the discontinuity of $C$ between $O$ and 1 . In practice, $C=0.5$ is used to characterize this surface.

\footnotetext{
${ }^{1}$ Université de Bordeaux, I2M, UMR 5295, F-33400 Talence, France.

${ }^{2}$ Université de Bordeaux, I2M, UMR 5295, F-33400 Talence, France.
} 
The resulting set of equations reads (Eqs. 1-4):

$$
\begin{gathered}
\nabla \cdot \boldsymbol{u}=0 \\
\rho\left(\frac{\partial \boldsymbol{u}}{\partial t}+(\boldsymbol{u} \cdot \nabla) \boldsymbol{u}\right)=\rho \boldsymbol{g}-\nabla p+\nabla \cdot\left(\left(\mu+\mu_{T}\right)\left(\nabla \boldsymbol{u}+\nabla^{t} \boldsymbol{u}\right)\right)+\boldsymbol{F}-\frac{\mu}{K} \boldsymbol{u} \\
\frac{\partial C}{\partial t}+\boldsymbol{u} \cdot \nabla C=0
\end{gathered}
$$

with velocity $\boldsymbol{u}$ and pressure $p$, assuming $\boldsymbol{g}$ as the gravity vector, $\rho$ as the density, $\mu$ as the viscosity, $\mu_{T}$ as the turbulent viscosity, $t$ as the time and $\boldsymbol{F}$ as the superficial tension volume force.

The magnitudes of the physical characteristics of the fluids are defined according to $C$ in a continuous manner as:

$$
\begin{aligned}
& \rho=C \rho_{1}+(1-C) \rho_{0} \\
& \mu=C \mu_{1}+(1-C) \mu_{0}
\end{aligned}
$$

where $\rho_{0}, \rho_{l}, \mu_{0}$ and $\mu_{l}$ are the densities and viscosities of fluid 0 and 1 respectively.

To deal with solid obstacles within the numerical domain, it is possible to use multi-grid domains, but it is often much simpler to implement the Brinkman theory, then considering the numerical domain as a unique porous medium. The permeability coefficient $K$ defines the capability of a porous medium to let pass the fluids more or less freely through it. A real porous medium is modeled with intermediate values of $K$. If this permeability coefficient is great $(K \rightarrow+\infty)$, the medium is equivalent to a fluid. If it is nil, we can model an impermeable solid. It is then possible to model moving rigid boundaries or complex geometries. To take this coefficient $K$ into account in our system of equations, we thus add an extra term (Eq. 2), called Darcy term, $(\mu / K) \boldsymbol{u}$.

Large scale turbulence is described by solving the flow equations (Eqs. 1 and 2), the small scale turbulence, which is not resolved by the flow model, is taken into account through a subgrid scale model. To represent the dissipative effect of the small turbulent structures, a turbulent viscosity $\mu_{T}$ is calculated with the Mixed Scale model (Sagaut 1998). We use the procedure of wave generation developed by Lin and Liu (1999). The method consists in introducing an internal mass source function in the continuity equation (Eq. 1) for a chosen group of cells defining the source region. The method has been extensively verified and validated compared with analytical profiles to ensure accurate wave generation.

Model (Eqs. 1 to 4) describes the entire hydrodynamics and geometrical processes involved in the motion of multiphase media.

\section{Numerical methods}

The time discretization is implicit and the equations are discretized on a staggered grid thanks to the finite volume method. The velocity/pressure coupling is solved with a pressure correction method (Goda 1978), which consists in splitting the Navier-Stokes system into two stages, a velocity prediction and a pressure correction. The space derivatives of the inertial term are discretized by a hybrid Upwind-Centered scheme and the viscous term is approximated by a second order centered scheme. The MPI library is used to parallelize the code. The MPI library is used to parallelize the code. The mesh is partitioned into equal size subdomains to ensure load balancing. Communications between processors are also minimized (Ahusborde and Glockner 2011).The HYPRE parallel solver and preconditioner library is used to solve the linear systems (Falgout et al. 2006). The prediction and correction steps are solved, respectively, thanks to a BiCGStab solver, associated with a point Jacobi preconditioner, and a GMRES solver, associated with a multigrid preconditioner. All the details are presented by Lubin et al. (2010).

\section{Interface description}

In this paper, we will present the numerical results obtained by using three different numerical methods for the free-surface description. 
The interface tracking is first achieved by a Volume Of Fluid method (VOF), which is able to handle interface reconnections without interface reconstruction (Lubin et al. 2006). The explicit Total Variation Decreasing (TVD) Lax-Wendroff (LW) scheme of LeVeque (1992) is used to solve directly the interface evolutions without the reconstruction of $C$ (Eq. 3). Then, we also used the classical Piecewise Linear Interface Construction (PLIC) VOF method of Youngs et al. (1982), which is able to handle interface reconnection with interface reconstruction to capture accurately strong interface distortions with large density and viscosity contrasts between fluids (Abadie et al. 1998). Considering the numerical difficulty and the large CPU cost induced to track every droplets and bubbles created in such flows (Deshpande et al., 2012), Vincent et al. (2010) proposed a hybrid Eulerian-Lagrangian numerical method (VOF-SM). The method is based on a Lagrangian sub-mesh description that employs Lagrangian markers to build a local Eulerian concentration. The interface tracking method allows a description of the multiphase flow at a scale smaller than the Eulerian grid cell (bubbles or droplets), with the usual Eulerian representation of the VOF presence function. The velocity field is discretized on a staggered grid, as usually. The computational domain is initially seeded with an equal number of markers in each computational cell. Each marker carries information such as volume and concentration. Once the Navier-Stokes equations have been solved on the Eulerian grid, the velocity is interpolated to advect the markers. Then, once the characteristics of the markers have been updated at the end of the transport step, the information is projected back to the Eulerian grid cells to reconstruct the macroscopic phase function $C$. The Lagrangian approach then allows an accurate and nondiffusive Eulerian description of the phase function $C$, for which the scales are smaller than the Eulerian space step (Vincent et al. 2010).

The accuracy of the numerical schemes and the conservation laws of mass and energy in the computational domain have been accurately verified. The numerical methods have already been fully described and extensively validated through numerous test cases including mesh refinement analysis (Lubin et al. 2006; Helluy et al. 2005; Lubin et al. 2010, 2011).

\section{NUMERICAL RESULTS}

After breaking near the shoreline, tsunami-wave trains form a sequence of turbulent bores propagating landward. These waves can also be seen collapsing upon nearshore breakwaters and generating violent impacts and overtopping flows. From a numerical point of view, the advantage of studying a single breaking wave is that the initial wave can be easily calculated thanks to analytical solutions. It also allows shorter CPU time simulations, as only one wave breaks. From the physical point of view, the wave breaking process and the evolution of the associated turbulent velocity field can be studied separately from the effect of return flow and residual turbulence. This would allow one to investigate the evolution of one breaking wave before tackling the more complicated problem involving a wave train. Many experimental data for solitary waves impinging and overtopping coastal structures are available in literature. These data can thus be used for further numerical model development and validation.

The numerical results presented hereafter were simulated with the VOF-TVD and the classical VOF-PLIC techniques, to first discuss results obtained with the most prevalent methods in literature. We will then show the capacity of the VOF-SM method to deal with the breaking of a solitary wave over a seawall.

\section{Description of the experimental configuration}

We investigated solitary waves impinging and overtopping an impermeable seawall on a 1:20 sloping beach, compared to the experimental results from Hsiao and Lin (2010). Three typical solitary wave cases with different breaking locations were studied:

- Solitary wave breaking before the coastal structure: a turbulent bore is generated and propagates landward, then impacts and overtops the seawall (Type 1).

- Solitary wave directly impacting on the seawall with overtopping flow subsequently generated (Type 2).

- Solitary wave overtopping upon the seawall crown and collapsing behind the structure (Type 3).

The experimental data showed detailed pictures of the free-surface deformations (overturning, splash-ups generation, air entrainment) and pressure recordings on the seawall. 


\section{Initial and boundary conditions}

To generate numerically the targeted solitary wave, we use the procedure developed by Lin and Liu (1999). The method consists of introducing an internal mass source function in the continuity equation (Eq. 1) for a chosen group of cells defining the source region:

$$
\nabla . \boldsymbol{u}=S(\boldsymbol{x}, t)
$$

where $S(\boldsymbol{x} ; t)$ is calculated thanks to any chosen analytical wave solution.

According to Lin and Liu (1999):

$$
S(\boldsymbol{x}, t)=\frac{2 c H}{A} \eta(t)
$$

where $A$ is the area of the source region, $c$ is the wave celerity, $d$ being the water depth, $H$ is the wave amplitude and $\eta(t)$ is the expected time history of free surface displacement (Lin and Liu 1999). Two wave trains of surface gravity waves are thus generated, as the free surface responds to a pressure increment defined within the source region cells. The area and the location of the source function have been designed applying the rules described by Lin and Liu (1999). The two identical wave trains propagate in opposite directions towards the both ends of the numerical domain. This method has already been implemented for the generation of regular waves breaking over a sloping beach (Lubin et al. 2011).

Other source wave maker methods can be found in the literature, but whatever the method used to generate the waves, the internal source is usually located somewhere in the numerical domain and a damping boundary condition has to be set at the outgoing boundary to let the waves propagate outside the numerical domain without numerical reflexion. In order to save some CPU time and use shorter numerical domains, we chose to set the source region at the left boundary, where we imposed a symmetry boundary condition (Fig. 1).

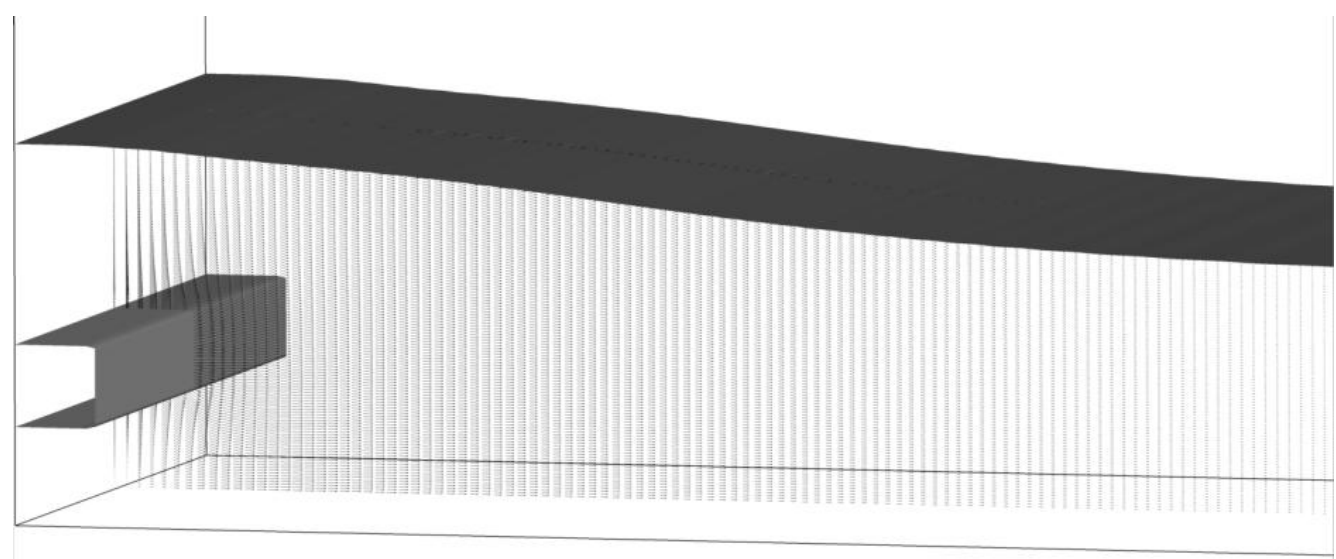

Figure 1. Solitary wave generated at the left boundary and propagating towards the right side of numerical domain. The velocity field is shown in the water responding to the increment of pressure due to the source function calculated in the horizontal rectangular area.

The three-dimensional computational domain is $8 \mathrm{~m}$ long, $2 \mathrm{~m}$ high and $0.5 \mathrm{~m}$ wide. The sloping beach starts at $x=0 \mathrm{~m}$, the source function being located at $x_{S}=0 \mathrm{~m}$ and $z_{S}=0.1 \mathrm{~m}$. The center of the source region is at $d / 2$, right above the toe of the sloping beach. The numerical beach is considered as an impermeable solid obstacle, the permeability coefficient $K$ being initialized at zero (Eq. 2). The source region is $0.1 \mathrm{~m}$ wide and $0.06 \mathrm{~m}$ high. The calculation is made with the densities and the viscosities of air and water $\left(\rho_{a}=1.1768 \mathrm{~kg} . \mathrm{m}^{-3}\right.$ and $\rho_{w}=1000 \mathrm{~kg} \cdot \mathrm{m}^{-3}, \mu_{a}=1.85 \times 10^{-5} \mathrm{~kg} \cdot \mathrm{m}^{-1} \cdot \mathrm{s}^{-1}$ and $\left.\mu_{w}=1 \times 10^{-3} \mathrm{~kg} \cdot \mathrm{m}^{-1} \cdot \mathrm{s}^{-1}\right)$. The $\mathrm{x}$-axis is taken parallel to the sloping beach, so the numerical domain is tilted off-vertical to match the bottom boundary with the bed slope. 


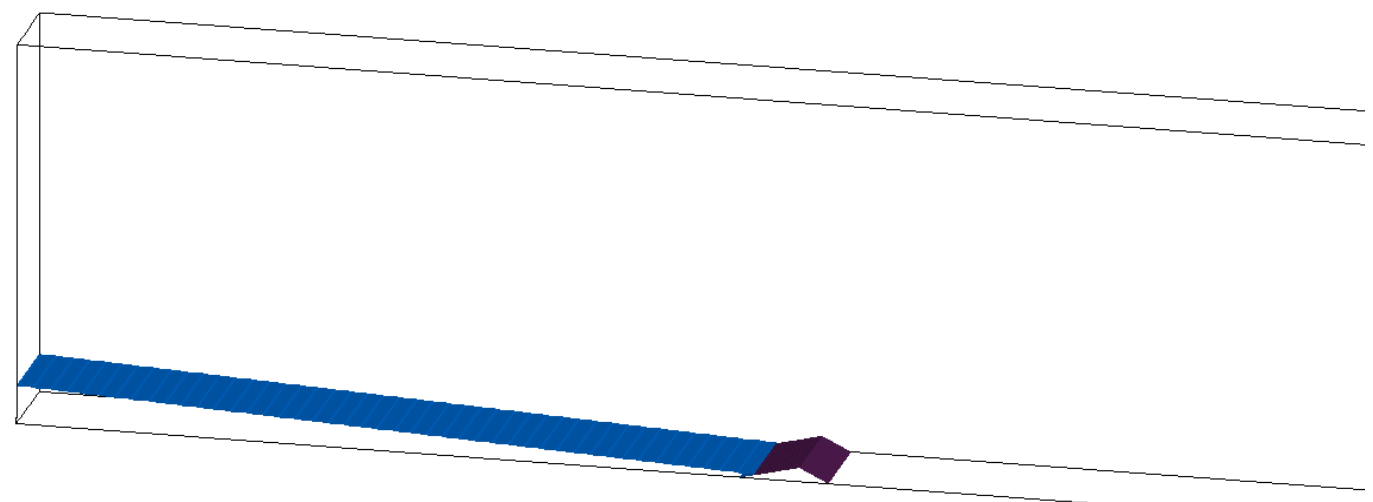

Figure 2. Sketch of the initial condition in the numerical wave tank, showing the initial free-surface identified with the isocontour of the colored function $C=0.5$. The seawall is located on the sloping beach.

Only two types of solitary waves are presented in this paper, the initial parameters experimented by Hsiao and Lin (2010) being given in Table 1.

\begin{tabular}{|l|c|c|}
\hline \multicolumn{3}{|c|}{ Table 1. Initial solitary wave parameters (Hsiao and Lin, 2010). } \\
\hline & Type 1 & Type 3 \\
\hline$d(m)$ & 0.2 & 0.256 \\
$H(m)$ & 0.07 & 0.0589 \\
$\varepsilon$ & 0.35 & 0.23 \\
\hline
\end{tabular}

\section{Results and discussion}

First, Type 1 solitary wave is simulated with a VOF-TVD scheme, as already used by Lubin et al. (2006, 2011). 5 millions mesh grid points are used to discretize the numerical domain, with uniform grids in each directions $\left(\Delta x_{\min }=8.10^{-3} \mathrm{~m}, \Delta z_{\min }=4.10^{-3} \mathrm{~m}, \Delta y_{\min }=5.10^{-2} \mathrm{~m}\right) .96$ processors have been used, one day being needed to simulate 16 seconds of physical time.

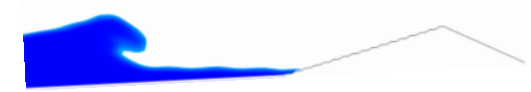

a)

c)

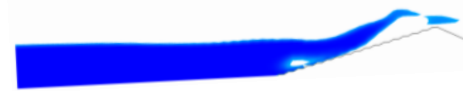

e)

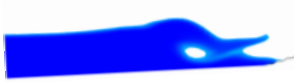

b)

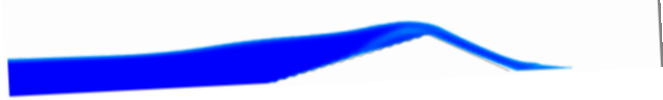

d)

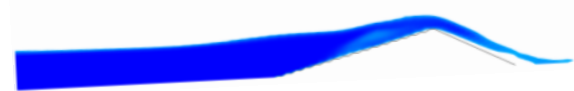

f)

Figure 3. Type 1 solitary wave breaking from a) to f). VOF-TVD method. $C \geq 0.5$. Slices taken in the middle of the 3D numerical domain. 
The main free-surface deformations are correctly simulated (Figs. 3). The results are qualitatively in agreement with the experimental pictures shown by Hsiao and Lin (2010). As the wave propagates over the slope of the beach, it looses its symmetry and the forward face of the wave becomes almost vertical. A jet of liquid is ejected from the crest and free falls down forward into a characteristic overturning motion. As expected, the wave breaks before the seawall structure. Then, the jet hits the forward face of the wave and closes over the air to form a main pocket of air. A series of subsequent splash-ups are observed. Due to the coarse mesh grid, only large pockets of air are entrained in the water, and no bubbles can be observed during the turbulent bore propagation.

The region of interest, where air and turbulence interacts, is also clearly affected by the numerical diffusion of the VOF-TVD scheme. Even if finer mesh grid cells are used, numerical diffusion will occur in strong mixing areas, which is the case in the turbulent bore region (Lubin et al. 2011). The overall behavior is qualitatively correct, but not satisfying concerning the air / water turbulent bore overtopping the seawall structure.

Then, the classical VOF-PLIC method has been used to overcome this limitation and increase the accuracy of the free-surface description. 20 millions mesh grid points are used to discretize the numerical domain, with uniform grids in each directions $\left(\Delta x_{\min }=1.10^{-4} \mathrm{~m}, \Delta z_{\min }=1.10^{-4} \mathrm{~m}, \Delta y_{\min }=\right.$ $\left.2.5 \times 10^{-2} \mathrm{~m}\right) .384$ processors have been used, 5 days being needed to simulate 3.4 seconds of physical time.

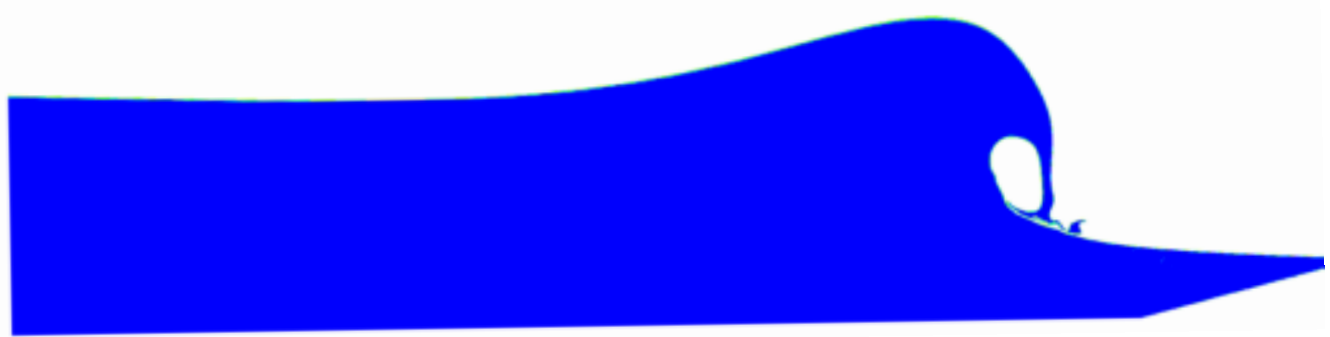

Figure 4. Type 1 solitary wave breaking. VOF-PLIC method. $C \geq 0.5$. Slices taken in the middle of the 3D numerical domain.

It can be clearly seen that the overturning wave is simulated much more accurately (Fig. 4). The plunge point is still located before the seawall structure, as expected. The plunging jet is much better described than with the VOF-TVD method. This implies that the volume of air entrapped by the plunging tongue of water is greater than previously simulated. This is in accordance with the experimental pictures shown by Hsiao and Lin (2010). The impacting jet is touching the forward face of the wave and starts to separate, one part forming the splash-up and the other wrapping around the main entrapped pocket of air. The tip of the jet is much more detailed with small inclusions of air and droplets being generated due to the fine mesh grid cells. But reaching this level of accuracy induces a large CPU cost to track every inclusions generated by the splashing jet several days for few seconds of physical time simulated).

Then, Type 3 has been simulated with the original VOF-SM method (Vincent et al. 2010). The Type 3 solitary wave involves less air entrainment. We first aim at testing the ability of the VOF-SM method to reproduce correctly the overtopping process. 5 millions Eulerian mesh grid points are used to discretize the numerical domain and 135 millions of Lagrangian markers, with uniform grids in each directions $\left(\Delta x_{\min }=8.10^{-3} \mathrm{~m}, \Delta z_{\min }=4.10^{-3} \mathrm{~m}, \Delta y_{\min }=5.10^{-2} \mathrm{~m}\right) .384$ processors have been used, 9 hours being needed to simulate 6 seconds of physical time.

The results are qualitatively in agreement with the experimental results, considering the large free-surface deformations (Figs. 5). More particles initialized in each Eulerian grid cells would improve the accuracy, but these first results are very encouraging and give a clear indication that the VOF-SM method is a numerical method which can provide a description of the multiphase flow at a scale smaller than the Eulerian grid cell. 


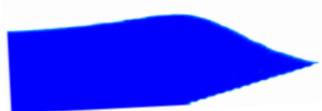

a)

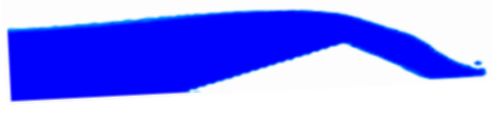

c)

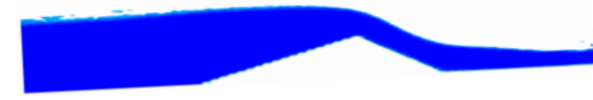

e)

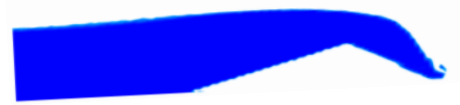

b)

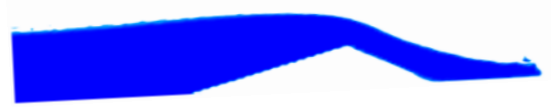

d)

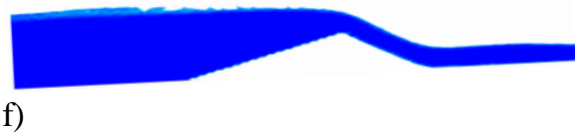

Figure 5. Type 3 solitary wave breaking. VOF-SM method. Eulerian color function reconstructed from the Lagrangian markers. $\mathrm{C} \geq 0.5$. Slices taken in the middle of the 3D numerical domain.

\section{CONCLUSIONS}

The numerical results presented in this paper concerns the free-surface deformations occurring when simulating three-dimensional breaking solitary waves impacting a seawall structure. The breaking process, in terms of wave overturning and splash-up occurrence, is in accordance with the general observations given in the literature. Our model was found to be able to describe correctly the complicated two-phase flow interactions that happen when waves break. The air entrainment can be described, which is important as it plays a great role in the energy dissipation process, but was shown to be dependant on the numerical methods used to track the free-surface as already discussed by Lubin et al. (2011). We then presented the first results of this on-going work, as the original VOF-SM method is now under intensive development. While the main features of the flow are in accordance with the experimental results and the method proved its ability to deal with strong interface distortions in an agitated flow, it still needs to be improved for some technical and physical aspects (Vincent et al. 2010). It is a first step towards subgrid physical modeling of multiphase flows involving LES turbulence models (Labourasse et al. 2007; Vincent et al. 2008). Mimicking the LES approach for turbulence, the VOF-SM method will be an appropriate method to take into account the inclusions smaller than the mesh grid size. Considering the difficulty to capture all the small bubbles and droplets encountered in the wave breaking problem, the small interface structures can thus be considered as subgrid interfaces.

\section{ACKNOWLEDGMENTS}

The authors wish to thank the Aquitaine Regional Council for the financial support towards a 256-processor cluster investment, located in the I2M laboratory. This work was granted access to the HPC resources of CINES under allocation 2012-x2012026104 made by GENCI (Grand Equipement National de Calcul Intensif). Computer time for this study was also provided by the computing facilities MCIA (Mésocentre de Calcul Intensif Aquitain) of the Université de Bordeaux and of the Université de Pau et des Pays de l'Adour. The authors also thank Dr. Hsiao and Dr. Lin for their valuable assistance and for kindly providing their experimental results. The development of the VOFSM method is financially supported by the French National Research Agency (Projet MODEMI ANR11-MONU-011-02). 


\section{REFERENCES}

Abadie, S., Caltagirone, J.-P. and Watremez, P. 1998. Mécanisme de génération du jet secondaire ascendant dans un déferlement plongeant, C. R. Mécanique, 326, 553-559.

Ahusborde, E. and S. Glockner. 2011. A 2d block-structured mesh partitioner for accurate flow simulations on non-rectangular geometries, Computers and Fluids, 43, 2-13.

Deshpande, S. S., M. F. Trujillo, X. Wu, and G. Chahine. 2012. Computational and experimental characterization of a liquid plunging into a quiescent pool at shallow inclination, International Journal of Heat and Fluid Flow, 34, 1-14.

Falgout, R. D., J. E. Jones, and U. M. Yang. 2006. The design and implementation of HYPRE, a library of parallel high performance preconditioners, chapter 51, 267-294. Numerical Solution of Partial Differential Equations on Parallel Computers. Springer-Verlag.

Helluy, P., F. Gollay, S. T. Grilli, N. Seguin, P. Lubin, J.-P. Caltagirone, S. Vincent, D. Drevard and R. Marcer. 2005. Numerical simulations of wave breaking. Mathematical Modelling and Numerical Analysis Mathematical Modelling and Numerical Analysis, 39 (3), 591-608.

Hsiao, S.-C. and T.-C. Lin. 2010. Tsunami-like solitary waves impinging and overtopping an impermeable seawall: Experiment and RANS modeling, Coastal Engineering, 57, 1-18.

Kataoka, I.. 1986. Local instant formulation of two-phase flow, International Journal of Multiphase Flow, 12 (5), 745-758.

Labourasse, E., D. Lacanette, A. Toutant, P. Lubin, S. Vincent, O. Lebaigue, J.-P. Caltagirone, and P. Sagaut. 2007. Towards Large Eddy Simulation of isothermal two-phase flows: governing equations and a priori tests. International Journal of Multiphase Flow, 33(1), 1-39.

LeVeque, R. J. 1992. Numerical methods for conservation laws, Lectures in Mathematics, Birkhauser, Zurich.

Lin, P., and P. L.-F. Liu. 1999. Internal wave-maker for Navier-Stokes equations models, Journal of Waterway, Port, Coastal and Ocean Engineering, 125, 322-330.

Lubin, P., S. Vincent, S. Abadie and J.-P. Caltagirone. 2006. Three-dimensional Large Eddy Simulation of air entrainment under plunging breaking waves, Coastal Engineering, 53, 631-655.

Lubin, P., H. Chanson, and S. Glockner. 2010. Large Eddy Simulation of turbulence generated by a weak breaking tidal bore, Environmental Fluid Mechanics, 10, 587-602.

Lubin, P., S. Glockner, O. Kimmoun, and H. Branger. 2011. Numerical study of the hydrodynamics of regular waves breaking over a sloping beach, European Journal of Mechanics B/Fluids, 30 (6), $552-564$.

Sagaut, P. 1998. Large eddy simulation for Incompressible Flows, Springer, Verlag.

Ting, F. 2008. Large-scale turbulence under a solitary wave: Part 2. Forms and evolution of coherent structures, Coastal Engineering, 55, 522-536.

Vincent, S., J. Larocque, D. Lacanette, A. Toutant, P. Lubin, and P. Sagaut. 2008. Numerical simulation of phase separation and a priori two-phase LES filtering. Computers and Fluids, 37, 898-906.

Vincent, S., G. Balmigère, J.-P. Caltagirone, and E. Meillot. 2010. Eulerian-Lagrangian multiscale methods for solving scalar equations - application to incompressible two-phase flows, Journal of Computational Physics, 229 (1), 73-106.

Youngs, D. L., K. W. Morton, and M. J. Baines. 1982. Time-dependent multimaterial flow with large fluid distortion, Numerical Methods for Fluid Dynamics. Academic Press, New York. 